\title{
openheart Improving the quality of care for patients with or at risk of atrial fibrillation: an improvement initiative in UK general practices
}

\author{
Yewande Adeleke (D) ," Dionne Matthew, ${ }^{1,2}$ Bradley Porter, ${ }^{3,4}$ Thomas Woodcock, ${ }^{1}$ \\ Jayne Yap, ${ }^{1,5}$ Sophia Hashmy, ${ }^{6}$ Ammu Mathew, ${ }^{7}$ Ron Grant, ${ }^{8}$ Agnes Kaba, ${ }^{7}$ \\ Brigitte Unger-Graeber, ${ }^{9}$ Sadia Khan, ${ }^{7}$ Derek Bell, ${ }^{2}$ Martin R Cowie ${ }^{10}$
}

\begin{abstract}
- Additional material is published online only. To view please visit the journal online (http://dx.doi.org/10.1136/ openhrt-2019-001086).
\end{abstract}

To cite: Adeleke $Y$, Matthew D, Porter B, et al. Improving the quality of care for patients with or at risk of atrial fibrillation: an improvement initiative in UK general practices. Open Heart 2019;6:e001086. doi:10.1136/ openhrt-2019-001086

This work has been previously presented as an oral abstract at the 2017 Heart Rhythm Congress. This abstract was based on preliminary data of number of newly diagnosed patients with atrial fibrillation over a shorter time period and using univariate analysis. De Vere F, Porter B, Woodcock $\mathrm{T}$, et al. Primary care based opportunistic screening for atrial fibrillation increases detection rates. Europace 2017;19:i6. doi: 10.1093/europace/eux283.023.

Received 8 May 2019

Revised 25 June 2019

Accepted 11 September 2019

Check for updates

(C) Author(s) (or their employer(s)) 2019. Re-use permitted under CC BY. Published by BMJ.

For numbered affiliations see end of article.

Correspondence to Yewande Adeleke; yewande. adeleke13@imperial.ac.uk

\section{ABSTRACT}

Objective Atrial fibrillation (AF) is a growing problem internationally and a recognised cause of cardiovascular morbidity and mortality. The London borough of Hounslow has a lower than expected prevalence of AF, suggesting poor detection and associated undertreatment. To improve AF diagnosis and management, a quality improvement (QI) initiative was set up in 48 general practices in Hounslow. We aimed to study whether there was evidence of a change in AF diagnosis and management in Hounslow following implementation of interventions in this QI initiative.

Methods Using the general practice information system (Systm0ne), data were retrospectively collected for 415626 patients, who were actively registered at a Hounslow practice between 1 January 2011 and 31 August 2018. Process, outcome and balancing measures were analysed using statistical process control and interrupted time series regression methods. The baseline period was from 1 January 2011 to 30 September 2014 and the intervention period was from 1 October 2014 to 31 August 2018.

Results When comparing the baseline to the intervention period, (1) the rate of new AF diagnoses increased by $27 \%$ (relative risk 1.27; 95\% $\mathrm{Cl} 1.05$ to 1.52; $\mathrm{p}<0.01$ ); (2) ECG tests done for patients aged 60 and above increased; (3) $\mathrm{CHA}_{2} \mathrm{DS}_{2}$-VASc and HAS-BLED risk assessments within 30 days of $A F$ diagnosis increased from $1.7 \%$ to $19 \%$ and $0.2 \%$ to $8.1 \%$, respectively; (4) among those at higher risk of stroke, anticoagulation prescription within 30 days of AF diagnosis increased from $31 \%$ to $63 \%$ while prescription of antiplatelet monotherapy within the same time period decreased from $17 \%$ to $7.1 \%$; and (5) average $\mathrm{CHA}_{2} \mathrm{DS}_{2}$ VASC and HAS-BLED risk scores did not change.

Conclusion Implementation of interventions in the Hounslow QI initiative coincided with improved AF diagnosis and management. Areas with perceived underdetection of AF should consider similar interventions and methodology.

\section{INTRODUCTION}

Atrial fibrillation (AF) is a common heart arrhythmia affecting 34 million people worldwide $^{1}$ and is associated with increased risk

\section{Key questions}

What is already known about this subject?

- Atrial fibrillation (AF) is a growing problem internationally and a recognised cause of cardiovascular morbidity and mortality. Despite published guidance, it is often undetected or undertreated in practice. Handheld ECG devices may facilitate screening of large numbers of individuals at risk of having this condition. Education of healthcare professionals and patients has been shown to increase appropriate oral anticoagulant prescription.

What does this study add?

This study found that use of quality improvement methodology to implement evidence-based interventions, including handheld ECG devices, may help improve AF diagnosis and management within the primary care setting.

How might this impact on clinical practice?

- These findings suggest that healthcare professionals working in areas with evidenced underdetection and undertreatment of AF should consider implementing similar interventions using quality improvement methodology.

of cardiovascular morbidity and mortality, including ischaemic stroke. ${ }^{2}$ The European Society of Cardiology (ESC) and the National Institute for Health and Care Excellence (NICE) have published guidelines for management of $\mathrm{AF}^{45}$ Despite this, $\mathrm{AF}$ is often undetected and undertreated. ${ }^{6}$

A multitude of factors contribute to poor implementation of AF guideline recommendations by healthcare professionals (HCP) ${ }^{7-9}$ To encourage implementation, many strategies have been employed. For instance, handheld ECG devices have been identified as feasible and efficient screening options for people at risk of developing $\mathrm{AF}^{10}{ }^{11} \mathrm{~A}$ systematic review identified that HCP and patient education 
significantly increased appropriate oral anticoagulant prescription, whereas computerised decision support tools and medication reviews did not. ${ }^{12}$ However, another study has shown that systematic identification and risk stratification of patients using electronic tools in conjunction with case note reviews and anticoagulation assessment clinics have improved $\mathrm{AF}$ diagnosis and management. ${ }^{13}$

In the UK, AF affects 1.2 million people ${ }^{14}$ and accounts for $1.0 \%$ of the total National Health Service (NHS) expenditure. ${ }^{15}$ Hounslow Clinical Commissioning Group (CCG) is one of eight CCGs in Northwest London (NWL), and comprises 48 general practices. ${ }^{16}$ In 2014, up to 2492 out of 292220 patients registered with a general practice in Hounslow borough had a diagnosis of AF, giving an observed prevalence of $0.9 \%,{ }^{17}$ compared with an expected prevalence of $1.6 \% .^{18}$ This suggests nearly 2000 people with undiagnosed and untreated AF. In the same year, $98.8 \%$ of patients diagnosed with $\mathrm{AF}$ were assessed for risk of stroke and $84.8 \%$ of patients diagnosed with $\mathrm{AF}$ with a $\mathrm{CHADS}_{2}$ score greater than 1 were prescribed anticoagulants. ${ }^{17}$ Despite these high proportions, there was a lack of information on timeliness of care from point of diagnosis. Furthermore, Hounslow's population aged over 65 is projected to increase by $18 \%$ by $2020,{ }^{19}$ this may have major implications for health service provision within the borough, as $\mathrm{AF}$ prevalence and incidence increases with age. ${ }^{1}$

In 2014, the Hounslow Quality Improvement (QI) team, supported by the National Institute for Health Research Collaboration for Leadership in Applied Health Research and Care NWL (CLAHRC NWL), began a QI initiative aiming to improve diagnosis and management of care for patients with or at risk of AF in Hounslow. This study aimed to evaluate the extent to which these aims were achieved.

\section{METHODS}

The intervention was a QI initiative comprising education and specialist support for general practitioners (GP) in diagnosis and management of $\mathrm{AF}$, alongside provision of KardiaMobile (AliveCor) handheld ECG devices to facilitate diagnosis.

\section{Study population}

The study population comprised patients registered at a general practice in Hounslow CCG prior to 31 August 2018, and who were still on the register by 31 August 2018 or ceased to be registered at some time after 1 January 2011. The latter is referred to as 'deduction' and occurs either through death or by deregistering, for example, moving out of Hounslow.

\section{Data extraction}

Pseudonymised data, in the form of Read-coded events, were retrospectively extracted from the SystmOne clinical general practice database on 10 September 2018. Data were extracted for the following events: registration with a GP practice, deduction from a practice list,
ECG screening, diagnosis of AF, risk scoring for stroke $\left(\mathrm{CHA}_{2} \mathrm{DS}_{2}\right.$-VASc) and bleeding (HAS-BLED) and prescription of anticoagulation. The Read code and event date were extracted, along with the age, gender and ethnicity of the patient, risk scores, and prescription drug, dose and duration. The data were cleaned to remove patients with missing or non-sensical registration dates $(n=3281)$, non-sensical deduction dates $(n=523)$, deduction dates preceding registration dates $(n=405)$ and non-sensical registration status $(n=66)$, some of these were duplicate patient records. A senior GP, with direct clinical responsibility for these patients, compared a sample of extracted data with patient records on SystmOne for data validation purposes. Data on AF diagnoses prior to 2011 could not be obtained from SystmOne. Instead, prevalence data were retrospectively collected from the Quality and Outcomes Framework (QOF) database for each year from 2011 to 2018.

\section{Measures}

The primary outcome of our investigation was the change in rate of $\mathrm{AF}$ diagnosis in Hounslow over time. The following outcome measures were assessed across the study population monthly: (1) proportion of patients with a new or pre-existing diagnosis of AF (prevalence); (2) number of patients newly diagnosed with AF; and (3) rate of new $\mathrm{AF}$ diagnosis. AF diagnoses were counted for all patients diagnosed with $\mathrm{AF}$ (including patients with an $\mathrm{AF}$ diagnosis alongside other comorbidities), irrespective of method of diagnosis such as 12-lead ECG, ambulatory ECG monitoring and KardiaMobile handheld ECG. There was no Read code specifically for the KardiaMobile device.

A 'risk register' of patients at high risk of AF was not available retrospectively, so overall numbers of ECG tests done in patients aged 60 and above were used as a proxy measure. Process measures after AF diagnosis measured four recommended elements of care: the proportion of patients who received a $\mathrm{CHA}_{2} \mathrm{DS}_{2}$-VASc risk assessment; proportion of patients who received a HAS-BLED risk assessment; proportion of indicated patients (ie, patients diagnosed with $\mathrm{AF}$ who have a $\mathrm{CHA}_{2} \mathrm{DS}_{2}$-VASc score of greater than or equal to 1 for males or greater than or equal to 2 for females) prescribed anticoagulation drug therapy; and proportion of indicated patients prescribed antiplatelet monotherapy. To understand the timeliness of the care provided we measured each proportion at 30, 90 and 180 days from AF diagnosis, along with the proportion who received the element in question at any point following diagnosis.

To understand whether efforts to increase AF diagnosis through screening inadvertently reached a subpopulation less at risk of stroke or bleeding than others, we measured the average $\mathrm{CHA}_{2} \mathrm{DS}_{2}$-VASc and HAS-BLED scores among patients newly diagnosed with AF. These measures acted as 'balancing measures'. ${ }^{20}$

\section{Statistical analysis}

Demographics of the study population, and the subpopulation with an $\mathrm{AF}$ diagnosis, were analysed as distributions 
of categorical variables age band, sex and ethnicity. Study population demographics were also evaluated at three time points: 31 December 2011; 31 December 2014; and 31 December 2017. Categorical variables are reported as total numbers and percentages and continuous variables as medians \pm IQR.

The baseline period, before the QI initiative began, was 1 January 2011 to 30 September 2014, and the intervention period was 1 October 2014 to 31 August 2018. Statistical process control (SPC) charts including $\mathrm{C}$ charts, $\mathrm{P}$ charts and $\bar{X}$ bar $\mathrm{S}$ control charts were created to understand the variation in the outcome, process and balancing measures over time and monitor the effect of changes. ${ }^{21}$ Established rules were used to determine whether variation was down to chance (online supplementary file 2). ${ }^{21}$ SPC analysis was performed using Microsoft Excel (V.1808).

To assess the rate of new AF diagnosis, an interrupted time series (ITS) regression was conducted. ${ }^{22} \mathrm{~A}$ level change model was selected, based on evidence that opportunistic screening using ECGs increases rates of AF diagnosis. ${ }^{23}$ An age and sex-standardised population was used to adjust for changes to population structures over time. $\mathrm{P}$ values $<0.05$ were deemed statistically significant. Quasi-Poisson regression was used to account for overdispersion in the data. The independent variables were time in months and the intervention. R statistical software (V.3.5.0) was used for the ITS analysis.

Pearson's $\chi^{2}$ test was used to compare whether indicated patients who have a HAS-BLED score were more likely to be anticoagulated than those who did not have a HAS-BLED score.

\section{QI initiative}

The QI team consisted of a consultant cardiologist, GP commissioner, cardiology nurses, project manager, CLAHRC NWL QI advisors and a patient with lived experience of AF. A systematic approach was used to guide evidence translation into practice,${ }^{24}$ making use of a range of improvement tools and methods, including the Model for Improvement, Stakeholder Mapping, Process Mapping, Action Effect Diagram ${ }^{25}$ and Plan-Do-Study-Act cycles. The intervention period was split into three phases (see online supplementary file 1 for the corresponding timeline).

\section{Interventions}

Phase I (October 2014 to January 2016)

An educational launch event was designed to improve HCPs' knowledge of AF diagnosis and management, and to highlight best practice. At this event, presentations were delivered by the QI team to representatives from all Hounslow general practices. The project manager demonstrated the KardiaMobile ECG device and how to record findings in SystmOne. Following this, the project manager made ad hoc general practice visits, which comprised education of GPs, practice nurses and health visitors on the importance of early detection of
AF, available treatment options, how to use the handheld ECG device, the aim of the QI initiative and its measures of success. These visits were tailored to the practice, and individual need, and lasted approximately 1 hour. In addition, the team attended Hounslow CCG network meetings to discuss the QI initiative with representatives from Hounslow general practices.

\section{Phase II (February 2016 to June 2016)}

Two handheld ECG devices and two mobile devices were distributed to each of the five pilot general practices to facilitate screening for AF within GP consultations, diabetes clinics, NHS health checks, influenza jab clinics, and so on. Mobile devices were used to encourage communication between HCPs and the QI team, for instance, to address queries about abnormal readings, or about the devices. A guide for HCPs on how to use the handheld ECG devices and mobile devices was created by the project manager using Microsoft PowerPoint.

An AF at-risk register was developed by the consultant cardiologist and the lead GP. The inclusion criteria for the at-risk register were patients aged 60 and above or on at least one of four disease registers: coronary heart disease, chronic obstructive pulmonary disease, obstructive sleep apnoea or hypertension. These disease registers were chosen in view of the community services for diabetes and heart failure already in existence in the borough, and that these conditions alone gave an at-risk register of more than 50000 people. A SystmOne AF clinical template was adapted from Nene CCG to encourage evidence-based practice in Hounslow and to upskill GPs on AF diagnosis and management. Patients who met the $\mathrm{AF}$ at-risk register criteria were flagged to GPs by a patient status alert linking to the clinical template.

\section{Phase III (July 2016 to March 2017)}

Each of the 43 remaining general practices received two handheld ECG devices, two mobile devices and an AF QOF data report for their practice. The same set-up process was followed as in phase II.

The QI team collaborated with HCPs and patients to coproduce eleven $5 \mathrm{~min}$ long 'electronic postcards' (videos, one of which was in Punjabi) to improve patient understanding of AF during and after consultation. These videos consist of members of the cardiology team explaining their role and giving information about $\mathrm{AF}$, its causes, symptoms, diagnosis, treatment options for rate and rhythm control and common side effects, the referral pathway, follow-up process, the ambulatory care pathway and patient support groups. Two of the videos feature patients living with AF describing their experience of the condition, how it has affected their quality of life and the lifestyle changes they made to manage their AF.

For all Hounslow general practices, a cardiology nurse reviewed AF registers, focusing on exception-reported patients and patients on aspirin, and ran face-to-face anticoagulation clinics. 
Table 1 Demographic characteristics of the Hounslow study population and the subpopulation stratified by AF diagnosis

\begin{tabular}{|c|c|c|}
\hline Characteristic & $\begin{array}{l}\text { Study population } \\
(\%)\end{array}$ & $\begin{array}{l}\text { AF } \\
\text { subpopulation } \\
(\%)\end{array}$ \\
\hline \multicolumn{3}{|l|}{ Registration status } \\
\hline Current & $276239(66.5)$ & $1728(57.5)$ \\
\hline Deceased, deducted & $7763(1.9)$ & $520(17.3)$ \\
\hline Deducted & $131624(31.7)$ & $759(25.2)$ \\
\hline \multicolumn{3}{|l|}{ Sex } \\
\hline Female & $194019(46.7)$ & $1363(45.3)$ \\
\hline Male & $221482(53.3)$ & $1642(54.6)$ \\
\hline Other/unknown & $125(0.0)$ & $2(0.1)$ \\
\hline \multicolumn{3}{|l|}{ Age } \\
\hline $0-19$ & 88891 (21.4) & $6(0.2)$ \\
\hline $20-29$ & $66519(16.0)$ & $14(0.5)$ \\
\hline $30-39$ & 88967 (21.4) & $44(1.5)$ \\
\hline $40-49$ & $62116(14.9)$ & $103(3.4)$ \\
\hline $50-59$ & $46612(11.2)$ & 247 (8.2) \\
\hline $60-69$ & 30577 (7.4) & $562(18.7)$ \\
\hline $70-79$ & $18763(4.5)$ & $862(28.7)$ \\
\hline $80-89$ & $10158(2.4)$ & $909(30.2)$ \\
\hline 90-99 & $2852(0.7)$ & $251(8.3)$ \\
\hline $100+$ & $171(0.04)$ & $9(0.3)$ \\
\hline \multicolumn{3}{|l|}{ Ethnicity } \\
\hline Asian or Asian British & $118460(28.5)$ & $480(16.0)$ \\
\hline $\begin{array}{l}\text { Black/African/ } \\
\text { Caribbean/Black } \\
\text { British }\end{array}$ & 20877 (5.0) & $71(2.4)$ \\
\hline $\begin{array}{l}\text { Mixed/multiple ethnic } \\
\text { groups }\end{array}$ & $11215(2.7)$ & $31(1.0)$ \\
\hline White & 168017 (40.4) & 1958 (65.1) \\
\hline Other ethnic group & $14836(3.6)$ & $53(1.8)$ \\
\hline Unknown & $82221(19.8)$ & $414(13.8)$ \\
\hline
\end{tabular}

$\mathrm{AF}$, atrial fibrillation.

The Standards for Quality Improvement Reporting Excellence 2.0 guidelines were used as a framework for reporting findings from this QI initiative. ${ }^{26}$

\section{RESULTS}

\section{Characteristics of study population}

Of 48 Hounslow general practices, data were available from 47 using the SystmOne software, but were not available from one practice that used a different clinical software (Egton Medical Information Systems). The study population consisted of 415626 patients registered at these practices, of which 3007 had an AF diagnosis at any time point from 1 January 2011 to 10 September 2018 (table 1 ). The study population had a median age of $36 \pm 27$ years, whereas patients with an $\mathrm{AF}$ diagnosis were older with a median age of $77 \pm 17$ years. AF diagnosis was most common in patients aged 80-89 years old (30\%) and ethnically white $(65 \%)$; known risk factors for $\mathrm{AF}$ (table 1). Patient demographics remained similar over the duration of the study (online supplementary file 3 ).

\section{Outcome measures}

\section{AF prevalence and incidence}

Annual AF prevalence recorded in Hounslow QOF data increased by 0.24 percentage points from 2011 to 2018, with most of this increase $(0.20 \%)$ occurring between 2014 and 2018, this represents a $0.04 \%$ average annual increase in $\mathrm{AF}$ prevalence from 2014 to 2018 compared with $0.01 \%$ between 2011 and $2014 .{ }^{17}$

In our ITS model, there was a statistically significant increase in the monthly rate of new AF diagnosis in the intervention period, with an increase of $27 \%$ (relative risk (RR) 1.27 ; $95 \%$ CI 1.05 to 1.52 ; $\mathrm{p}<0.01$ ) (online supplementary file 4). Adjusting for seasonality did not alter this finding (RR: $1.24 ; 95 \%$ CI 1.04 to 1.48 ; $\mathrm{p}<0.01$ ). Autocorrelation was assessed, and the lags were found not to have a significant effect. This increase is also seen in the raw number of patients newly diagnosed with $\mathrm{AF}$, which increased from an average of 28 per month in the baseline period to 38 per month from March 2015, during phase I (figure 1).

\section{Process measures}

\section{ECG screening}

The number of ECG tests done for patients aged 60 years and above increased from an average of 98 per month in the baseline period to 135 per month from January 2015 (phase I) (figure 2). A large transient increase was observed from June 2016 (phase II), but this did not persist.

\section{$\mathrm{CHA}_{2} \mathrm{DS}_{2}$-VASc and HAS-BLED risk assessments}

The monthly proportion of patients with an AF diagnosis who received a $\mathrm{CHA}_{2} \mathrm{DS}_{2}-\mathrm{VASc}$ risk assessment within 30 days of AF diagnosis increased from an average of $1.7 \%$ in the baseline period to $10 \%$ from February 2015 (phase I), and further rose to 19\% from November 2016 (phase III) (figure 3). Similarly, increases were observed for the 90 days, 180 days and at any time from AF diagnosis measures (online supplementary file 5).

The monthly proportion of patients with an AF diagnosis who received a HAS-BLED risk assessment increased from an average of $0.2 \%$ in the baseline period to $8.1 \%$ from October 2014 (phase I) (figure 4). A transient increase was observed from September 2015 (phase I), but this did not persist. Similarly, increases were observed for the 90 days, 180 days and at any time from AF diagnosis measures (online supplementary file 5).

\section{Anticoagulation and antiplatelet drug therapy}

The monthly proportion of indicated patients prescribed anticoagulation drug therapy within 30 days of $\mathrm{AF}$ diagnosis increased from an average of $31 \%$ in 
Monthly number of patients newly diagnosed with AF (January 2011-August 2018)

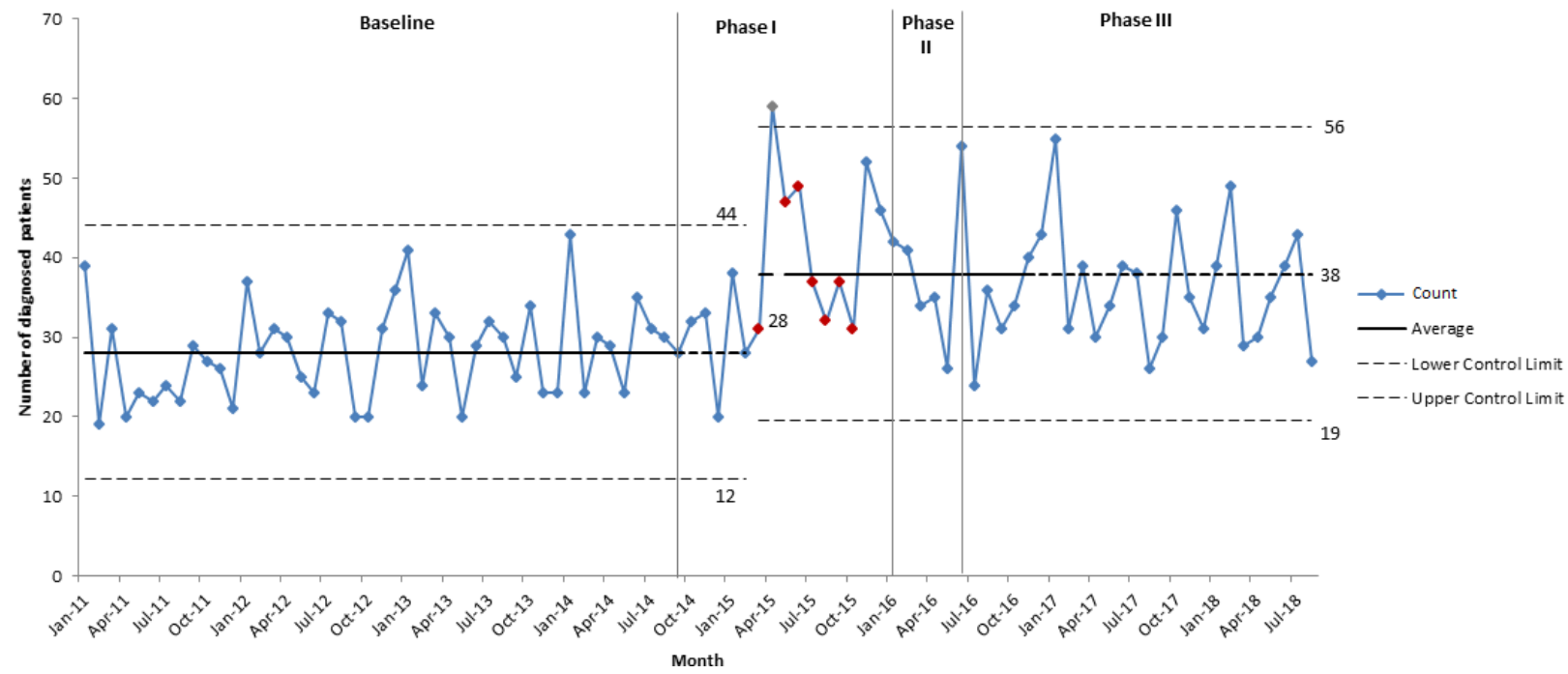

Figure 1 C-chart showing monthly number of patients newly diagnosed with AF, from January 2011 to August 2018 . The increase from 28 patients newly diagnosed with AF each month in the baseline period to 38 each month is clearly seen in phase I of the intervention period. AF, atrial fibrillation.

the baseline period to $55 \%$ from October 2014 (phase I) (figure 5). A further increase to $63 \%$ is observed in phase III of the intervention period. Similarly, increases were observed for the 90 days, 180 days and at any time from AF diagnosis measures (online supplementary file 5).

Conversely, the monthly proportion of indicated patients prescribed antiplatelet monotherapy within 30 days of $\mathrm{AF}$ diagnosis decreased from an average of $17 \%$ in the baseline period to $7.1 \%$ from January 2015 (phase I) (figure 6). Similarly, decreases were observed for the 90 days, 180 days and at any time from AF diagnosis measures (online supplementary file 5).

\section{Balancing measures}

$\mathrm{CHA}_{2} \mathrm{DS}_{2}-\mathrm{VASc}$ and HAS-BLED risk scores

The monthly average $\mathrm{CHA}_{2} \mathrm{DS}_{2}$-VASc score in the baseline period was 3.4 and this remained the same throughout the intervention period (online supplementary file 6). Similarly, average HAS-BLED risk

Monthly number of ECG tests done for patients aged 60 and above at time of screening (January 2011 - August 2018)

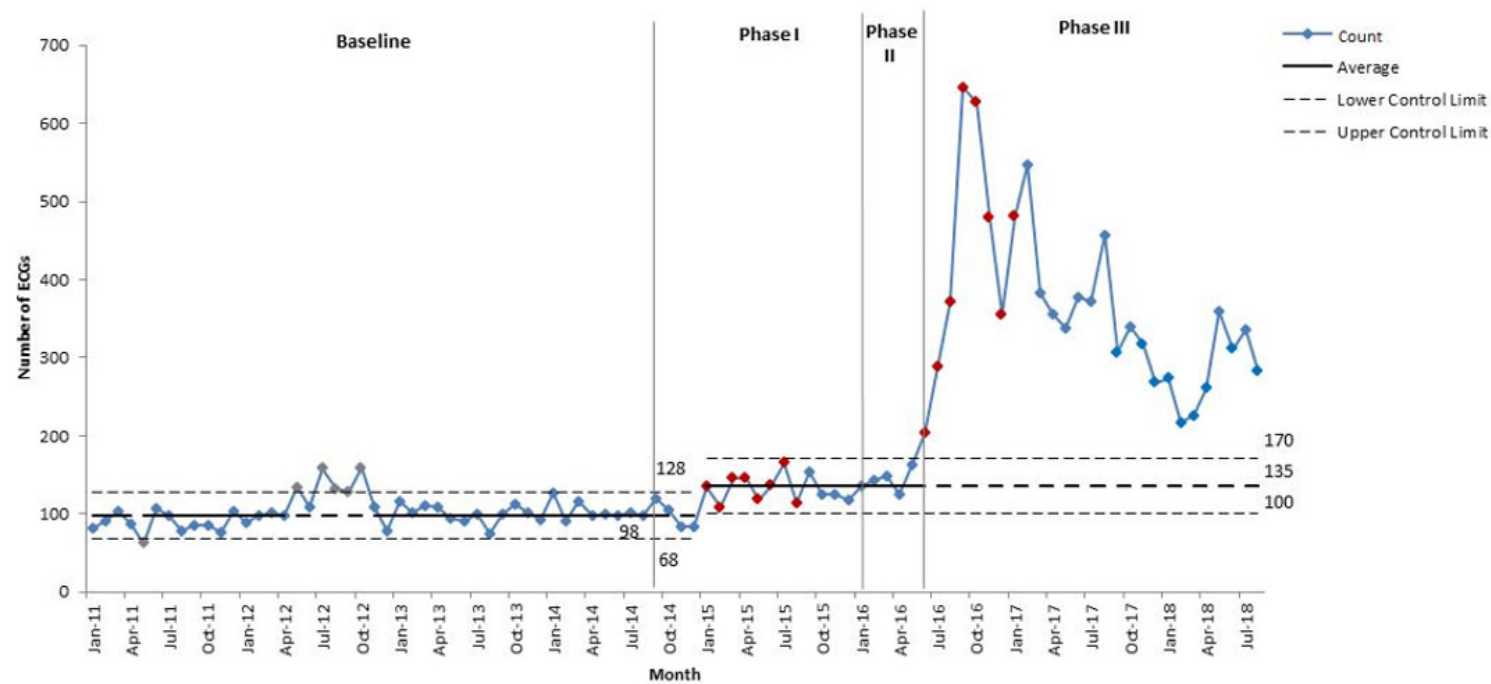

Figure 2 C-chart showing monthly number of ECG tests done for patients aged 60 and above at time of screening, from January 2011 to August 2018. The increase from 98 ECG tests done per month in the baseline period to 135 ECGs done is clearly seen in phase I of the intervention period. Although a further increase is observed in phase III of the intervention period, this increase was unstable, thus the control limits and centre line have not been revised. 
Monthly proportion of patients with an AF diagnosis who received a CHA2DS2-VASc risk assessment within 30 days of AF diagnosis

(January 2011- July 2018)

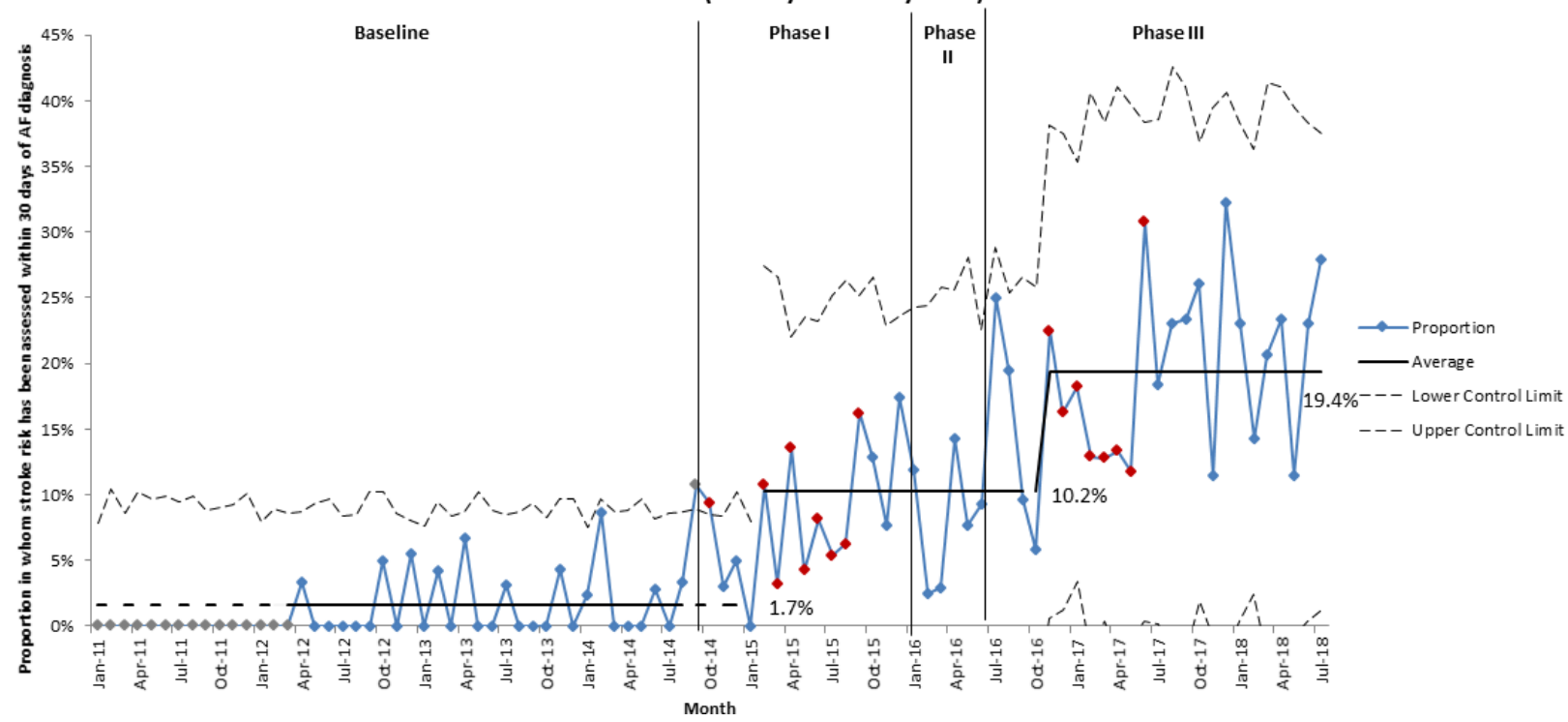

Figure 3 P-chart showing monthly proportion of patients with an AF diagnosis who received a $\mathrm{CHA}_{2} \mathrm{DS}_{2}-\mathrm{VASc}$ risk assessment within 30 days of AF diagnosis, from January 2011 to July 2018 . The increase from $1.7 \%$ of patients with an AF diagnosis who received a $\mathrm{CHA}_{2} \mathrm{DS}_{2}$-VASc risk assessment within 30 days of AF diagnosis in the baseline period to $10 \%$ is clearly seen in phase I of the intervention period. A further increase to $19 \%$ is observed in phase III of the intervention period. AF, atrial fibrillation.

scores in the intervention period did not change from the baseline average of 1.6 (online supplementary file 7$)$.

Indicated patients who have a HAS-BLED score are more likely to be anticoagulated than those who do not have a HAS-BLED score $(\mathrm{p}<0.001)$.

\section{DISCUSSION Principal findings}

Between baseline and intervention periods, the number of ECG tests conducted per month for patients aged 60 and above increased. Diagnosed incidence and prevalence of $\mathrm{AF}$ also increased, although prevalence remained lower

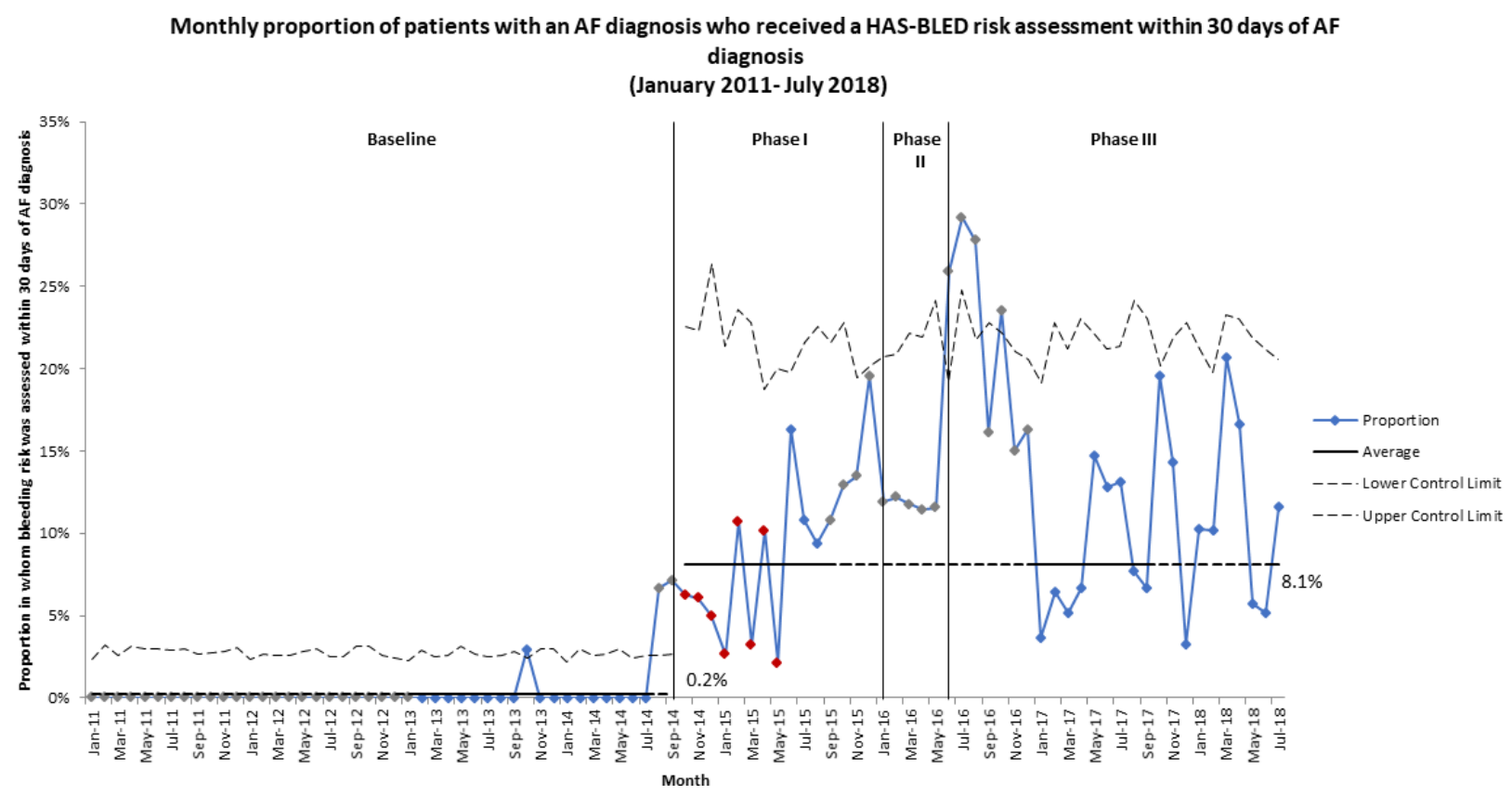

Figure 4 P-chart showing monthly proportion of patients with an AF diagnosis who received a HAS-BLED risk assessment within 30 days of AF diagnosis, from January 2011 to July 2018 . The increase from $0.2 \%$ of patients with an AF diagnosis who received a HAS-BLED risk assessment within 30 days of AF diagnosis in the baseline period to $8.1 \%$ is clearly seen in phase I of the intervention period. AF, atrial fibrillation. 
Monthly proportion of indicated patients prescribed anticoagulation therapy within 30 days of AF

diagnosis

(January 2011- July 2018)

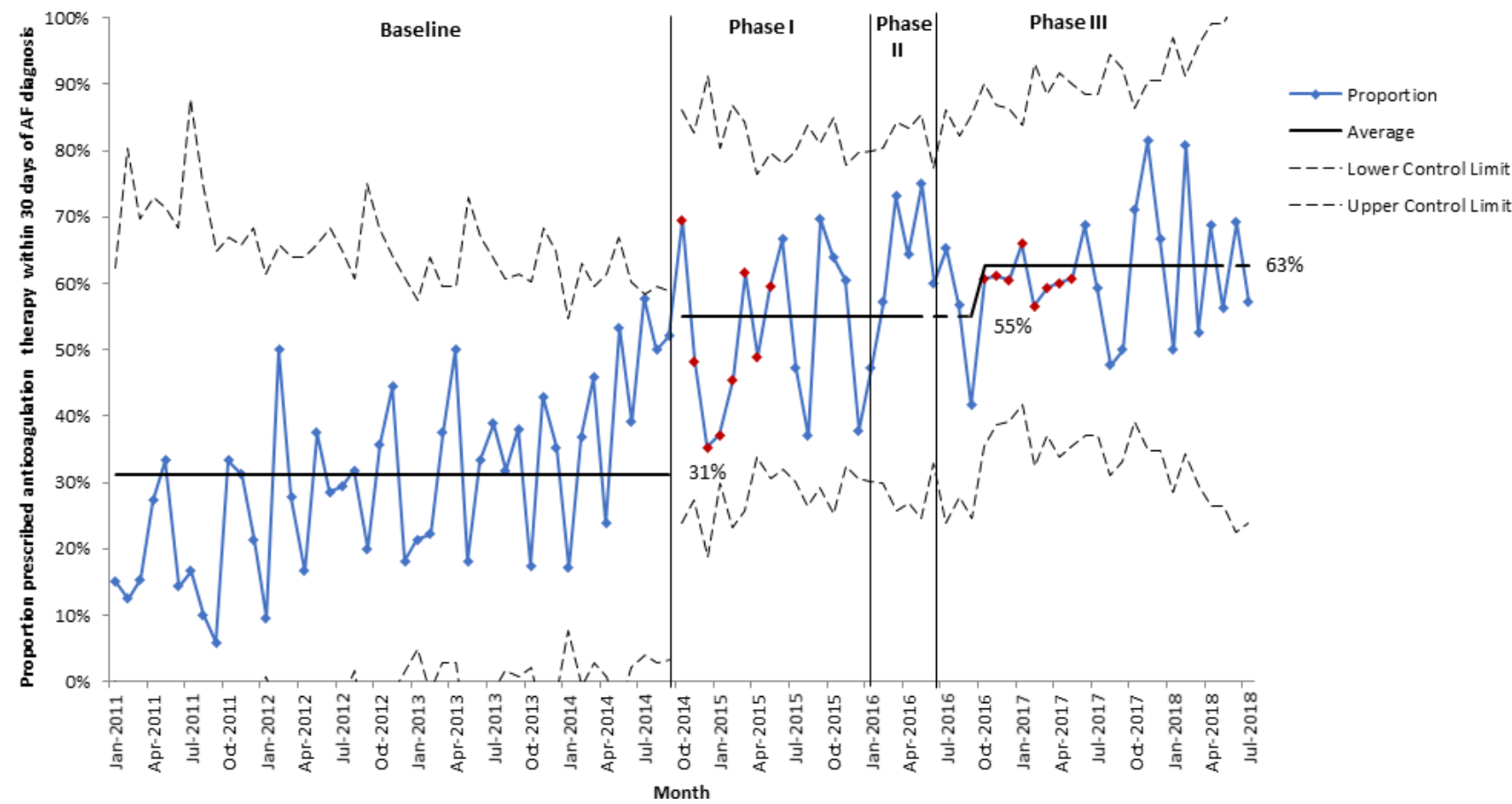

Figure 5 P-chart showing monthly proportion of indicated patients prescribed anticoagulation treatment within 30 days of AF diagnosis, from January 2011 to July 2018. The increase from $31 \%$ of indicated patients prescribed anticoagulation treatment within 30 days of AF diagnosis in the baseline period to $55 \%$ is clearly seen in phase I of the intervention period. A further increase to $63 \%$ is observed in phase III of the intervention period. AF, atrial fibrillation.

than the level expected for the population. Within those newly diagnosed with $\mathrm{AF}$, the proportion who received timely $\mathrm{CHA}_{2} \mathrm{DS}_{2}-\mathrm{VASc}$ and HAS-BLED risk assessments increased, and risk profiles did not change. A greater proportion of patients indicated for anticoagulation received appropriate prescriptions, while prescription of antiplatelet monotherapy decreased. Despite these promising improvements in measures related to management of $\mathrm{AF}$, there remains room for improvement in provision of timely care. This is supported by the fact that, on lifting the time limit (ie, 30/90/180 days from AF diagnosis) for these measures, the average proportion of patients receiving best practice elements of care increases (online supplementary file 5).

\section{ECG screening}

Increases in ECG screening suggest that a combination of HCP education, availability of handheld ECG devices, an $\mathrm{AF}$ at-risk register and an $\mathrm{AF}$ clinical template with a linked patient status alert is a viable means of increasing opportunistic screening in primary care. However, increases were unstable, and the possible decline from September 2017 may indicate that further HCP engagement is necessary, or that following an initial increase in diagnoses, the remaining undiagnosed population is still not being reached.

\section{AF prevalence and incidence}

AF prevalence and incidence increased during the intervention period. Demographic factors remained relatively unchanged between 2011 and 2017, indicating that the increases were not due to a changing proportion of frail elderly. There was a steady population increase in Hounslow between 2011 and 2018 in contrast with the step change in new AF diagnoses. This suggests that the increase in AF diagnosis rate is not simply a reflection of increasing population. Although we cannot assume causality, it is possible that HCP education may have contributed to the increase in the number and rate of new AF diagnosis. However, increased AF prevalence and incidence may be associated with other secular trends such as national focus on $\mathrm{AF}$ and anticoagulation (eg, $\mathrm{AF}$ awareness week) or introduction of the NICE guidelines on AF diagnosis and management in June 2014. After the initial increase of new AF diagnosis in March 2015, there were no further increases observed. This indicates that the distribution of handheld ECG devices from June 2016 onwards and the accompanying increase in screening did not result in higher case detection. A possible reason is that at-risk but ultimately healthy patients were being screened-for example, there may be participation bias where population characteristics differ between general practice attenders and non-attenders, in terms of healthseeking behaviours and health status. 
Monthly percentage of indicated patients prescribed antiplatelet drug therapy within 30 days of AF

diagnosis

(January 2011-July 2018)

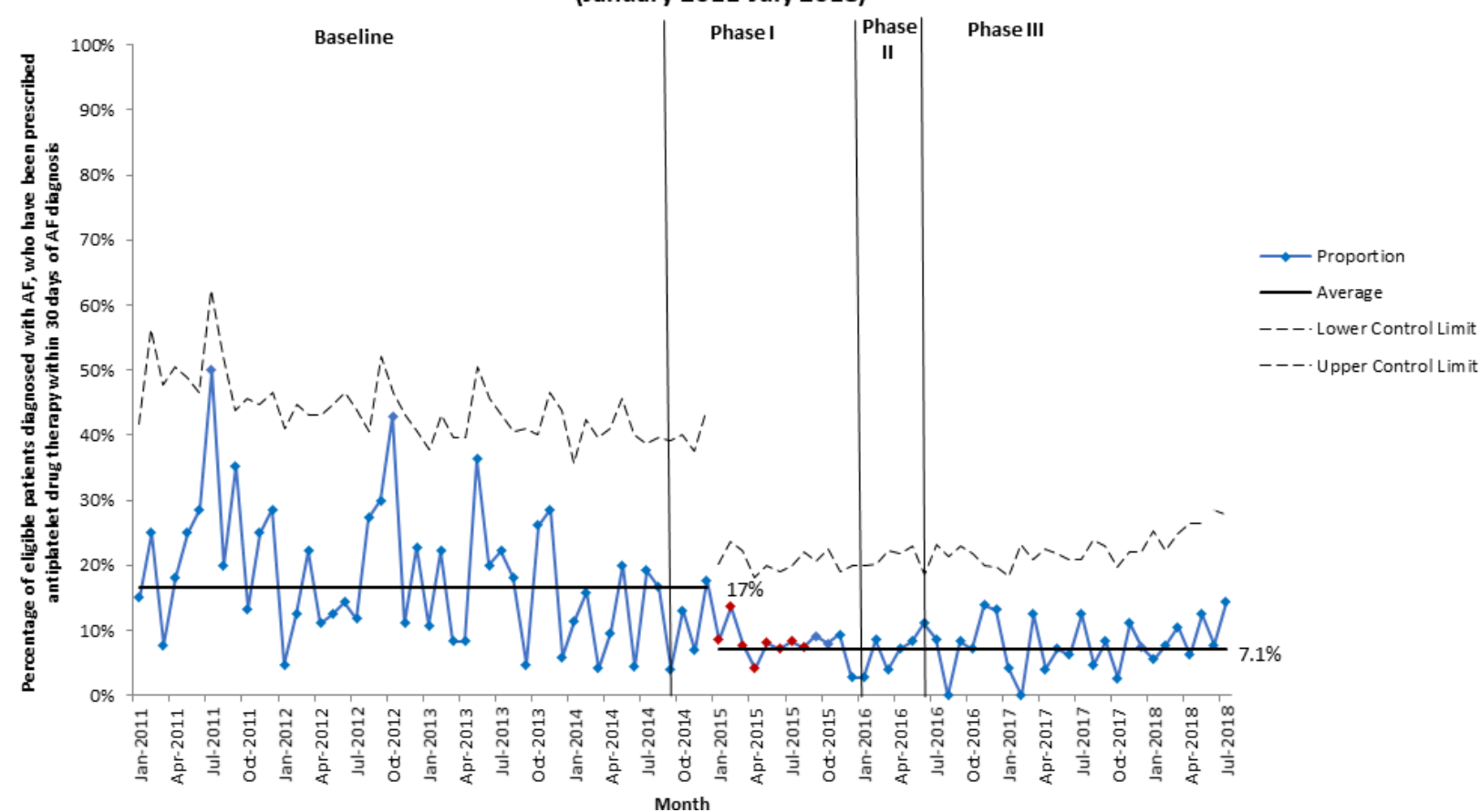

Figure 6 P-chart showing monthly proportion of indicated patients prescribed antiplatelet monotherapy within 30 days of AF diagnosis, from January 2011 to July 2018. The decrease from $17 \%$ of indicated patients who were prescribed antiplatelet monotherapy within 30 days of AF diagnosis in the baseline period to $7.1 \%$ is clearly seen in phase I of the intervention period. $\mathrm{AF}$, atrial fibrillation.

\section{$\mathrm{CHA}_{2} \mathrm{DS}_{2}$-VASC and HAS-BLED risk assessments}

Improvements in stroke and bleeding risk assessments for patients with an AF diagnosis occurred during the intervention period. It is plausible that increases in risk assessments after June 2014 are associated with the updated NICE guidelines, at least at the start of the intervention period. Further increases may be due to the QI initiative. Nonetheless, improved timeliness of risk assessments over time is indicative of increased clinical activity towards mitigating AF-related stroke risk.

Although guidelines state that bleeding risk should be taken into consideration for patients diagnosed with $\mathrm{AF}$ to identify and treat risk factors, the proportion of patients in whom bleeding risk was assessed was lower than that of stroke risk assessments. Despite increases in bleeding risk assessments during QI initiative's active funding period (October 2015 to March 2017), these increases were not sustained. The lower proportion of bleeding risk assessments could be due to these risk assessments historically not being done in routine practice for patients diagnosed with $\mathrm{AF}^{27}$ or may be because bleeding risk assessments unlike stroke risk assessments are not nationally incentivised in the QOF. During the initiative, the QI team were careful to emphasise the 2016 ESC guideline position: 'A high bleeding risk score should generally not result in withholding oral anticoagulation. Rather, bleeding risk factors should be identified and treatable factors corrected. ${ }^{4}$

\section{Anticoagulation drug therapy and antiplatelet monotherapy}

Anticoagulation prescription in patients for whom it was indicated increased during the intervention period, with a decrease in antiplatelet monotherapy. Improvements in these measures may be due to the QI initiative but as previously mentioned causality cannot be attributed on the basis of this study. Despite these improvements, the proportion of indicated patients receiving anticoagulants within 30 days is still quite low. Reasons for this could include retrospective or delayed data recording in SystmOne or patient choice. It seems unlikely that bleeding risk played a major role in this, however, based on the result that indicated patients scored for bleeding are more likely to be prescribed anticoagulation than those not scored.

A small proportion of patients with an AF diagnosis are still on aspirin monotherapy despite guideline recommendations against its prescription for stroke prevention in patients diagnosed with $\mathrm{AF}^{10}$ This may be due to patient preferences or aspirin monotherapy prescription for another comorbidity, but also suggests that further work needs to be undertaken around this.

\section{$\mathrm{CHA}_{2} \mathrm{DS}_{2}$-VASC and HAS-BLED risk scores}

Stable $\mathrm{CHA}_{2} \mathrm{DS}_{2}$-VASc and HAS-BLED risk scores in the intervention period means that the increased screening did not lead to detection of AF in patients with a different risk profile when compared with the baseline period. 


\section{Comparison with existing literature}

Findings from this study concur with previous studies showing HCP education, clinical decision support tools and screening can contribute to improved $\mathrm{AF}$ diagnosis and management. ${ }^{12}{ }^{1328-30}$ Studies have tested the feasibility and accuracy of using handheld ECG devices in AF screening programmes in different settings, by various users, for both systematic and opportunistic screening approaches. ${ }^{10}$ 31-33 All report acceptable rates of newly diagnosed $\mathrm{AF}$ and positive perceptions towards the technology. This research goes one step further focusing on implementation of this device in routine care.

\section{Strengths and limitations}

Use of routinely collected primary care data from all but one Hounslow general practice minimises selection bias, and including both outcome and process measures provide a thorough assessment of $\mathrm{AF}$ diagnosis and management pathways.

It is difficult to know if improvements were associated with the interventions, wider secular trends, or unmeasured confounders such as guidelines, reimbursement, and so on, because there is no control group. The QI initiative may have only temporarily changed GPs' behaviours, as suggested by the decline in some measures towards the end of the study. Retrospective data on the AF at-risk population were unavailable, hence ECG tests done for patients aged 60 years and above were used as a proxy measure for ECGs done within the AF at-risk population. Using the available data, it was not possible to distinguish between ECG tests and AF diagnoses resulting directly from use of the KardiaMobile device, and those resulting from traditional ECG screening. AF diagnoses were only available from 2011 onwards, hence, there may be patients with unknown $\mathrm{AF}$ within the study population. The proportion of $\mathrm{CHA}_{2} \mathrm{DS}_{2}$-VASc risk assessments in the baseline period may not be a true representation of stroke risk assessments done, as the older, less comprehensive $\mathrm{CHADS}_{2}$ risk assessment tool was used prior to NICE recommending use of the $\mathrm{CHA}_{2} \mathrm{DS}_{2}$-VASc risk assessment tool ${ }^{34}$ and also patients with an $\mathrm{AF}$ diagnosis may still be prescribed anticoagulants at a clinician's discretion without having done a $\mathrm{CHA}_{2} \mathrm{DS}_{2}-\mathrm{VAS}_{\mathrm{C}}$ risk assessment or the risk assessment may be done but not recorded. Finally, population and practice characteristics of Hounslow could limit generalisation of the findings.

\section{Implications for future research}

Further research should focus on the reasons behind the remaining gaps between expected and observed prevalence, including developing more subtle prediction models and understanding prevalence in subpopulations. It is not clear to what extent the interventions deployed in the Hounslow QI initiative are suitable to reveal further undiagnosed prevalence, and further improve risk scoring and anticoagulation, or whether additional interventions may be required to achieve this. The longterm impact of this initiative is not clear from the data available for this study, future research should look at hospital admissions for stroke and bleeding with history of AF, as well as HCP and patient perceptions of the QI initiative, as well as barriers and facilitators to sustaining the observed gains.

\section{CONCLUSION}

Using electronic health record data, this study found that implementation of synergistic evidence-based interventions in a QI initiative coincided with improvements in $\mathrm{AF}$ diagnosis and management in 47 general practices in NWL. It has highlighted the disparity between quality of care as measured by QOF data and more granular analysis including timeliness of patient care. Other healthcare areas with perceived underdetection of AF should consider similar interventions and methodology to improve AF diagnosis and management.

\section{Author affiliations}

${ }^{1}$ Department of Primary Care and Public Health, Imperial College London, Chelsea and Westminster Hospital, NIHR Collaboration for Leadership in Applied Health Research and Care for Northwest London, London, UK

${ }^{2}$ Chelsea and Westminster Hospital, Chelsea and Westminster Hospital NHS Foundation Trust, London, UK

${ }^{3}$ Cardiology Department, Guy's and St Thomas' NHS Foundation Trust, London, UK ${ }^{4}$ St Thomas' Hospital, King's College London, London, UK

${ }^{5}$ Clinical Quality, Performance and Technology, Government of Singapore Ministry of Health, Outram, Singapore

${ }^{6}$ North West London Clinical Commissioning Groups, London, UK

${ }^{7}$ Cardiology Department, West Middlesex University Hospital, Chelsea and

Westminster Hospital NHS Foundation Trust, London, UK

${ }^{8}$ Upbeat Heart Prevention and Support Group, London, UK

${ }^{9}$ Chiswick Health Practice, London, UK

${ }^{10}$ National Heart and Lung Institute, Imperial College London, London, UK

Acknowledgements The authors thank the following people for their valuable contributions: Adrian Adams, Kwame Agyapong, Gabriel Burcea, Jake Clements, Linda Eftychiou, Meerat Kaur, Derryn Lovett, Rachel Mak, Mable Nakubulwa, Raffaele Palladino, Tom Rollinson, Shaheeda Saiyed and David Sunkersing.

Contributors SK and BUG conceived the idea for the study. All the authors contributed to the design and planning of the study. YA, DM and TW performed statistical analysis. YA wrote the first draft of the manuscript. All authors revised the manuscript critically and have given their final approval of the version to be published. YA and DM are responsible for the overall content.

Funding This article presents independent research in part funded by the National Institute for Health Research (NIHR) under the Collaboration for Leadership in Applied Health Research and Care (CLAHRC) programme for Northwest London (NWL). This study was supported in part by a Medical and Education Goods and Services (MEGS) grant from Pfizer and Hounslow CCG provided technology (ie, KardiaMobile (AliveCor) and mobile devices)

Disclaimer The views expressed in this publication are those of the author(s) and not necessarily those of the NHS, the NIHR or the Department of Health and Social Care.

Competing interests MRC reports grants and personal fees from Bayer, personal fees from Pfizer BMS Alliance, outside the submitted work. SK reports grants from Pfizer, during the conduct of the study.

Patient consent for publication Not required.

Ethics approval According to policy on activities that constitute research at West Middlesex University Hospital NHS Trust, this work met criteria for operational improvement activities and is exempt from ethics review. Ethical approval was not required for this work as it was part of a service evaluation and improvement activity and not human subjects research. An ethics waiver was granted by West Middlesex University Hospital NHS Trust research executive. 
Provenance and peer review Not commissioned; internally peer reviewed.

Data availability statement All data relevant to the study are included in the article or uploaded as supplementary information.

Open access This is an open access article distributed in accordance with the Creative Commons Attribution 4.0 Unported (CC BY 4.0) license, which permits others to copy, redistribute, remix, transform and build upon this work for any purpose, provided the original work is properly cited, a link to the licence is given, and indication of whether changes were made. See: https://creativecommons.org/ licenses/by/4.0/.

ORCID iD

Yewande Adeleke http://orcid.org/0000-0002-4357-9855

\section{REFERENCES}

1 Chugh SS, Havmoeller R, Narayanan K, et al. Worldwide epidemiology of atrial fibrillation. Circulation 2014;129:837-47.

2 Odutayo A, Wong CX, Hsiao AJ, et al. Atrial fibrillation and risks of cardiovascular disease, renal disease, and death: systematic review and meta-analysis. BMJ 2016;354.

3 Wolf PA, Abbott RD, Kannel WB. Atrial fibrillation as an independent risk factor for stroke: the Framingham study. Stroke 1991;22:983-8.

4 Kirchhof P, Benussi S, Kotecha D, et al. Esc guidelines for the management of atrial fibrillation developed in collaboration with EACTS. Eur Heart J 2016;2016:2893-962.

5 National Institute for Health and Care Excellence. Atrial fibrillation: management. Available: https://www.nice.org.uk/guidance/cg180/ [Accessed 8 Apr 2019].

6 Lip GYH, Kakar P, Watson T. Atrial fibrillation--the growing epidemic. Heart 2007;93:542-3.

7 Di PG LR. New atrial fibrillation guidelines: implementation in the clinic. Clin Pract 2013;10:31-8.

8 Mant J, Fitzmaurice DA, Hobbs FDR, et al. Accuracy of diagnosing atrial fibrillation on electrocardiogram by primary care practitioners and interpretative diagnostic software: analysis of data from screening for atrial fibrillation in the elderly (safe) trial. BMJ 2007;335:380-2.

9 Taggar JS, Coleman T, Lewis S, et al. Screening for atrial fibrillation a cross-sectional survey of healthcare professionals in primary care. PLoS One 2016;11:e0152086-14.10.1371/journal.pone.0152086

10 Ramkumar S, Nerlekar N, D'Souza D, D'Souza D, et al. Atrial fibrillation detection using single lead portable electrocardiographic monitoring: a systematic review and meta-analysis. BMJ Open 2018;8:e024178.

11 Fay MR, Fitzmaurice DA, Freedman B. Screening of older patients for atrial fibrillation in general practice: current evidence and its implications for future practice. Eur J Gen Pract 2017;23:246-53.

12 Pritchett RV, Bem D, Turner GM, et al. Improving the prescription of oral anticoagulants in atrial fibrillation: a systematic review. Thromb Haemost 2019;119:294-307.

13 Das M, Panter L, Wynn GJ, et al. Primary care atrial fibrillation service: outcomes from consultant-led anticoagulation assessment clinics in the primary care setting in the UK. BMJ Open 2015;5:e009267.

14 Quality and Outcomes Framework. QOF database UK atrial fibrillation. Available: https://www.gpcontract.co.uk/browse/UK/Atria Fibrillation/18 [Accessed 8 Apr 2019].

15 Stewart S, Murphy N, Walker A, et al. Cost of an emerging epidemic: an economic analysis of atrial fibrillation in the UK. Heart 2004;90:286-92.

16 Hounslow CCG. About us, 2019. Available: https://www. hounslowccg.nhs.uk/about-us.aspx [Accessed 8 Apr 2019].
17 Quality and Outcomes Framework. QOF database NHS HOUNSLOW CCG atrial fibrillation. Available: https://www.gpcontract.co.uk/ browse/07Y/Atrial Fibrillation/11 [Accessed 8 Apr 2019].

18 Public Health England. Atrial fibrillation prevalence estimates. Available: https://www.gov.uk/government/publications/atrialfibrillation-prevalence-estimates-for-local-populations [Accessed 8 Apr 2019].

19 North West London collaboration of clinical Commisioning groups. Hounslow population demographics. Available: https://www.heal thiernorthwestlondon.nhs.uk/services/hounslow [Accessed $29 \mathrm{Apr}$ 2019].

20 Toma M, Dreischulte T, Gray NM, et al. Balancing measures or a balanced accounting of improvement impact: a qualitative analysis of individual and focus group interviews with improvement experts in Scotland. BMJ Qual Saf 2018;27:547-56.

21 Provost LP, Murray SK. The health care data guide: learning from data for improvement. First. Jossey-Bass 2011.

22 Lopez Bernal J, Cummins S, Gasparrini A. Interrupted time series regression for the evaluation of public health interventions: a tutorial. Int J Epidemiol 2016;12:dyw098.

23 Fitzmaurice DA, Hobbs FDR, Jowett S, et al. Screening versus routine practice in detection of atrial fibrillation in patients aged 65 or over: cluster randomised controlled trial. BMJ 2007;335.

24 Reed JE, Howe C, Doyle C, et al. Successful healthcare improvements from translating evidence in complex systems (SHIFTEvidence): simple rules to guide practice and research. Int J Qual Heal Care 2018:1-7.

25 Reed JE, McNicholas C, Woodcock T, et al. Designing quality improvement initiatives: the action effect method, a structured approach to identifying and articulating programme theory. BMJ Qual Saf 2014;23:1040-8.

26 Ogrinc G, Davies L, Goodman D, et al. Squire 2.0 (standards for quality improvement reporting excellence): revised publication guidelines from a detailed consensus process. BMJ Qual Saf 2016:25:986-92.

27 Pisters R, Lane DA, Nieuwlaat R, et al. A novel user-friendly score (HAS-BLED) to assess 1-year risk of major bleeding in patients with atrial fibrillation. Chest 2010;138:1093-100.

28 Robson J, Dostal I, Mathur R, et al. Improving anticoagulation in atrial fibrillation: observational study in three primary care trusts. $\mathrm{Br} \mathrm{J}$ Gen Pract 2014;64:e275-81.

29 Eckman MH, Lip GYH, Wise RE, et al. Impact of an atrial fibrillation decision support tool on thromboprophylaxis for atrial fibrillation. Am Heart J 2016;176:17-27.

30 Orchard J, Neubeck L, Freedman B, et al. eHealth tools to provide structured assistance for atrial fibrillation screening, management, and Guideline-Recommended therapy in metropolitan general practice: the AF-SMART study. J Am Heart Assoc 2019;8:e010959.

31 Orchard J, Lowres N, Freedman SB, et al. Screening for atrial fibrillation during influenza vaccinations by primary care nurses using a smartphone electrocardiograph (iECG): a feasibility study. Eur J Prev Cardiol 2016;23(2_suppl):13-20.

32 Lowres N, Neubeck L, Salkeld G, et al. Feasibility and costeffectiveness of stroke prevention through community screening for atrial fibrillation using iPhone ECG in pharmacies. The SEARCH-AF study. Thromb Haemost 2014;111:1167-76.

33 William AD, Kanbour M, Callahan T, et al. Assessing the accuracy of an automated atrial fibrillation detection algorithm using smartphone technology: the iREAD study. Heart Rhythm 2018;15:1561-5.

34 Yarmohammadi H, Varr BC, Puwanant S, et al. Role of CHADS2 score in evaluation of thromboembolic risk and mortality in patients with atrial fibrillation undergoing direct current cardioversion (from the acute trial substudy). Am J Cardiol 2012;110:222-6. 